\title{
CRITICAL LIEB-THIRRING BOUNDS IN GAPS AND THE GENERALIZED NEVAI CONJECTURE FOR FINITE GAP JACOBI MATRICES
}

RUPERT L. FRANK and BARRY SIMON

\section{Abstract}

We prove bounds of the form

$$
\sum_{e \in I \cap \sigma_{\mathrm{d}}(H)} \operatorname{dist}\left(e, \sigma_{\mathrm{e}}(H)\right)^{1 / 2} \leq L^{1} \text {-norm of a perturbation, }
$$

where $I$ is a gap. Included are gaps in continuum one-dimensional periodic Schrödinger operators and finite gap Jacobi matrices, where we get a generalized Nevai conjecture about an $L^{1}$-condition implying a Szegó condition. One key is a general new form of the Birman-Schwinger bound in gaps.

\section{Introduction}

This paper discusses spectral theory of Schrödinger operators, $-\Delta+V$ on $L^{2}\left(\mathbb{R}^{v}\right)$, and Jacobi matrices

$$
J=\left(\begin{array}{cccc}
b_{1} & a_{1} & 0 & \ldots \\
a_{1} & b_{2} & a_{2} & \ldots \\
0 & a_{2} & b_{3} & \ldots \\
\vdots & \vdots & \vdots & \ddots
\end{array}\right)
$$

on $\ell^{2}\left(\mathbb{Z}_{+}\right)$.

One of the streams motivating our work here is critical Lieb-Thirring inequalities. For any self-adjoint operator, $A$, define

$$
S^{\gamma}(A)=\sum_{e \in \sigma_{\mathrm{d}}(A)} \operatorname{dist}\left(e, \sigma_{\mathrm{e}}(A)\right)^{\gamma},
$$

where $\sigma_{\mathrm{d}}$ is the discrete spectrum and $\sigma_{\mathrm{e}}$ the essential spectrum, and the sum counts any $e$ the number of times of its multiplicity. Then the original Lieb-Thirring bounds 
(see [39]) assert that (here $\left.V_{-}=\max (0,-V)\right)$

$$
S^{\gamma}(-\Delta+V) \leq L_{\gamma, v} \int V_{-}(x)^{\gamma+v / 2} d^{\nu} x
$$

for a universal constant, $L_{\gamma, v}$. In [39], Lieb and Thirring proved this for $\gamma>1 / 2$ if $v=1$ and for $\gamma>0$ if $v \geq 2$. The endpoint result for $\gamma=0$ if $v \geq 3$ is the celebrated Cwikel-Lieb-Rosenblum (CLR) bound (see [30], [37] for reviews and history of LiebThirring and related bounds). For $v=1$, the endpoint result (called the critical bound) for $\gamma=1 / 2$ is due to Weidl [54], with an alternate proof and optimal constant due to Hundertmark, Lieb, and Thomas [31].

Here we are interested in analogues of the critical bound in one dimension for perturbations of operators other than $-\Delta$. For perturbations of the free Jacobi matrix ( $J$ with $b_{n} \equiv 0, a_{n} \equiv 1$ ), the critical bound is due to Hundertmark and Simon [32], and for perturbations of periodic Jacobi matrices to Damanik, Killip, and Simon [19]. In [22], Frank, Simon, and Weidl proved bounds of the form

$$
\sum_{\substack{e<\inf \sigma\left(H_{0}\right) \\ e \in \sigma(H)}} \operatorname{dist}\left(e, \sigma\left(H_{0}\right)\right)^{1 / 2} \leq c \int|V(x)| d x
$$

for $H_{0}=-\frac{d^{2}}{d x^{2}}+V_{0}$ and proved the Jacobi analogue for $e<\inf \sigma\left(J_{0}\right)$ and $e>$ $\sup \sigma\left(J_{0}\right)$, where $H_{0}$ has a regular ground state and, in particular, in the case of periodic $V_{0}$.

Typical of our new results is the following.

\section{THEOREM 1.1}

Let $V_{0}$ be a periodic, locally $L^{1}$-function on $\mathbb{R}$. Let $(a, b)$ be a gap in the spectrum of $H_{0}=-\frac{d^{2}}{d x^{2}}+V_{0}$. Then there is a constant $c$ so that for any $V \in L^{1}(\mathbb{R})$, one has

$$
\sum_{\substack{e \in \sigma_{\mathrm{d}}\left(H_{0}+V\right) \\ e \in(a, b)}} \operatorname{dist}\left(e, \sigma\left(H_{0}\right)\right)^{1 / 2} \leq c \int|V(x)| d x .
$$

\section{Remark}

This is an analogue of a result of Damanik, Killip, and Simon [19] for perturbations of periodic Jacobi matrices; they used what they call the magic formula to reduce to a critical Lieb-Thirring bound for matrix perturbations of a free Jacobi matrix. They have a magic formula for periodic Schrödinger operators, but it yields a nonlocal unperturbed object for which there is no obvious Lieb-Thirring bound. 
The other stream motivating this work goes back to a conjecture of Nevai [41, p. 92] that if a Jacobi matrix, $J$, obeys

$$
\sum_{n=1}^{\infty}\left|a_{n}-1\right|+\left|b_{n}\right|<\infty
$$

then its spectral measure,

$$
d \rho(x)=f(x) d x+d \rho_{\mathrm{s}}(x)
$$

(with $d \rho_{\mathrm{s}}$ singular), obeys a Szegó condition

$$
\int_{-2}^{2}\left(4-x^{2}\right)^{-1 / 2} \log (f(x)) d x>-\infty .
$$

This conjecture was proven by Killip and Simon [34].

THEOREM 1.2 (Killip and Simon [34, Theorem 2, p. 257])

(1.6) implies (1.8).

Their method, the model for analogues, is in two parts.

(a) Prove a theorem that

$$
\prod_{n=1}^{N} a_{n} \rightarrow 1
$$

plus

$$
\sum_{e \in \sigma_{d}(J)} \operatorname{dist}\left(e, \sigma_{e}(J)\right)^{1 / 2}<\infty
$$

implies (1.8). This generalizes results of Szegő, Shohat, and Nevai (see [49] for the history).

(b) Prove a critical Lieb-Thirring bound (in this case, done by Hundertmark and Simon [32]) to prove that (1.6) implies (1.10).

Since (1.6) clearly implies (1.9), we get (1.8). This strategy was exploited by Damanik, Killip, and Simon [19] to prove an analogue of Nevai's conjecture for perturbations of periodic Jacobi matrices. Here we are interested in a larger class called finite gap Jacobi matrices. Let $\mathfrak{e}$ be a closed subset of $\mathbb{R}$ whose complement has $\ell$ open intervals plus two unbounded pieces: $\mathfrak{e}=\mathfrak{e}_{1} \cup \cdots \cup \mathfrak{e}_{\ell+1}$ and $\mathfrak{e}_{j}=\left[\alpha_{j}, \beta_{j}\right]$ with $\alpha_{1}<\beta_{1}<\alpha_{2}<\cdots<\alpha_{\ell+1}<\beta_{\ell+1}$. Periodic Jacobi matrices have $\sigma_{\mathrm{e}}(J)$ equal to such an $\mathfrak{e}$, where each $\mathfrak{e}_{j}$ has rational harmonic measure, so such $\mathfrak{e}$ 's are a small subset 
of all finite gap $\mathfrak{e}$ 's. In such a case, the set of periodic Jacobi matrices with $\sigma_{\mathrm{e}}(J)=\mathfrak{e}$ is a torus of dimension $\ell$. For general $\mathfrak{e}$ 's, there is still a natural $\ell$-dimensional isospectral torus of almost periodic $J$ 's with $\sigma_{\mathrm{e}}(J)=\mathfrak{e}$. It is described, for example, in [17].

Here is another main result of this paper.

\section{THEOREM 1.3}

Let $\left\{a_{n}^{(0)}, b_{n}^{(0)}\right\}_{n=1}^{\infty}$ be the Jacobi parameters for an element of the isospectral torus of a finite gap set, e. Let $\left\{a_{n}, b_{n}\right\}$ be a set of Jacobi parameters obeying

$$
\sum_{n=1}^{\infty}\left|a_{n}-a_{n}^{(0)}\right|+\left|b_{n}-b_{n}^{(0)}\right|<\infty .
$$

Then the spectral measure, $d \rho$, of this perturbed Jacobi matrix has the form (1.7), where

$$
\int_{\mathfrak{e}} \operatorname{dist}(x, \mathbb{R} \backslash \mathfrak{e})^{-1 / 2} \log (f(x)) d x>-\infty
$$

One part of our proof involves the general theory of eigenvalues in gaps, a subject with considerable literature (see [1], [2], [5]- [15], [23], [25]- [28], [33], [35], [38], [45]-[47], [50], [51]). We will find a general Birman-Schwinger-type bound that could also be used to simplify many of these earlier works. To describe this bound, we make several definitions.

If $C$ is self-adjoint and $I \subset \mathbb{R}$ and $I \cap \sigma_{\mathrm{e}}(C)=\emptyset$, we define

$$
N(C \in I)=\operatorname{dim}\left(\operatorname{Ran}\left(P_{I}(C)\right)\right)
$$

with $P_{I}(\cdot)$ a spectral projection. We have $N(C>\alpha)=N(C \in(\alpha, \infty))$.

Recall that if $A$ is a self-adjoint operator bounded from below, a quadratic form $B$ is called relatively $A$-compact if $Q(A) \subset Q(B)$, and for $e<\inf \sigma(A),(A-$ $e)^{-1 / 2} B(A-e)^{-1 / 2}$ is compact; that is, for some compact operator $K$ and all $u, v \in \mathcal{H}$,

$$
B\left((A-e)^{-1 / 2} u,(A-e)^{-1 / 2} v\right)=(u, K v) .
$$

Often, $B$ is also an operator, in which case we may refer to an operator being form compact. The Birman-Schwinger principle says that if $B_{-} \geq 0$ is relatively $A$-compact and $E<\inf \sigma(A)$, then (see [30])

$$
N\left(A-B_{-}<E\right)=N\left(B_{-}^{1 / 2}(A-E)^{-1} B_{-}^{1 / 2}>1\right) .
$$

There is a slight abuse of notation in (1.14) since a form need not have a square root. We need to suppose that our positive forms, $B$, can be written $C^{*} C$, where $C: \mathscr{H}_{+1} \rightarrow \mathcal{K}$ with $\mathscr{H}_{+1}, \mathscr{H}_{-1}$ the usual scale of spaces (see [44]) and $\mathcal{K}$ an arbitrary 
space (usually $\mathcal{K}=\mathscr{H}) \cdot B^{1 / 2}(A-E)^{-1} B^{1 / 2}$ is then $C(A-E)^{-1} C^{*}$. We call a form of this type factorizable when $C$ is compact as a map from $\mathscr{H}_{-1}$ to $\mathcal{K}$. In our examples, since either $B$ is bounded and $C=\sqrt{B}$ or $B$ is multiplication by $f \geq 0$ with $f \in L^{1}$ and $C=$ multiplication by $\sqrt{f}$, we use the simpler notation.

Suppose that $E \notin \sigma(A)$ and $B \geq 0$ is relatively compact. As $x$ varies from zero to 1 , the discrete eigenvalues of $A \pm x B$ are analytic in $x$ and strictly monotone, so there are only finitely many such $x$ 's for which $E \in \sigma(A \pm x B)$. We define $\delta_{ \pm}(A, B ; E)$ to be the number of solutions (counting multiplicity) with $x$ in $(0,1)$. Equation (1.14) is proven by noting that

$$
N\left(A-B_{-}<E\right)=\delta_{-}\left(A, B_{-} ; E\right)
$$

and

$$
\delta_{-}\left(A, B_{-} ; E\right)=N\left(B_{-}^{1 / 2}(A-E)^{-1} B_{-}^{1 / 2}>1\right) .
$$

Prior approaches to eigenvalues in gaps rely on going from $A$ to $A+B$ via $A \rightarrow A+B_{+} \rightarrow A+B_{+}-B_{-}$or via $A \rightarrow A-B_{-} \rightarrow A+B_{+}-B_{-}$. Thus, for example, by the same argument that leads to (1.15),

$$
\begin{aligned}
N\left(A+B_{+}-B_{-} \in(\alpha, \beta)\right)= & \delta_{+}\left(A, B_{+} ; \alpha\right)-\delta_{+}\left(A, B_{+} ; \beta\right) \\
& +\delta_{-}\left(A+B_{+}, B_{-} ; \beta\right)-\delta_{-}\left(A+B_{+}, B_{-} ; \alpha\right) .
\end{aligned}
$$

The analogues of (1.16) for $B \geq 0$ are

$$
\begin{aligned}
& \delta_{-}(A, B ; E)=N\left(B^{1 / 2}(A-E)^{-1} B^{1 / 2}>1\right), \\
& \delta_{+}(A, B ; E)=N\left(B^{1 / 2}(A-E)^{-1 / 2} B^{1 / 2}<-1\right) .
\end{aligned}
$$

Dropping the negative terms in (1.17) leads to

$$
\begin{aligned}
N(A+B \in(\alpha, \beta)) \leq N & \left(B_{+}^{1 / 2}(A-\alpha)^{-1} B_{+}^{1 / 2}<-1\right) \\
& +N\left(B_{-}^{1 / 2}\left(A+B_{+}-\beta\right)^{-1} B_{-}^{1 / 2}>1\right) .
\end{aligned}
$$

The $B_{+} B_{-}$-cross-terms in (1.20) make it difficult to get Lieb-Thirring-type bounds although, with the other results of this paper, one could prove Theorem 1.3 from (1.20). What allows us to get Lieb-Thirring bounds is the following improvement of (1.20) that has no cross-terms.

\section{THEOREM 1.4}

Let $B_{+}$and $B_{-}$be nonnegative, relatively form compact, factorizable perturbations of a semibounded self-adjoint operator, A. Let $[\alpha, \beta] \subset \mathbb{R} \backslash \sigma(A)$. Suppose $\alpha, \beta \notin$ 
$\sigma\left(A+B_{+}\right) \cup \sigma\left(A-B_{-}\right) \cup \sigma\left(A+B_{+}-B_{-}\right)$. Then

$$
\begin{aligned}
N\left(A+B_{+}-B_{-} \in(\alpha, \beta)\right) \leq & N\left(B_{+}^{1 / 2}(A-\alpha)^{-1} B_{+}^{1 / 2}<-1\right) \\
& +N\left(B_{-}^{1 / 2}(A-\beta)^{-1} B_{-}^{1 / 2}>1\right) .
\end{aligned}
$$

\section{Remarks}

(1) $B_{+}, B_{-}$need not be the positive and negative part of a single operator; in particular, they need not commute.

(2) While it is not stated as a formal theorem and not applied, Pushnitski [42] mentions (1.21) explicitly (following [42, Corollary 3.2]).

We prove this result in Section 2. We use this in Section 3 to prove a CLR bound for perturbations of $-\Delta+V_{0}$, where $V_{0}$ is a putatively generic periodic potential in $\mathbb{R}^{v}, v \geq 3$. Section 4 provides an abstract result that shows that if there is an eigenfunction expansion near a gap, with eigenfunctions smooth in a parameter $k$ with energies quadratic in $k$, then a critical Lieb-Thirring bound holds at that gap edge. The proof will reduce to the original critical Lieb-Thirring bound, and so shed no light on why that bound holds. (We regard both proofs of that bound as somewhat miraculous; see [54], [31].) In Section 5, we apply the abstract theorem to periodic Schrödinger operator and so get Theorem 1.1, and in Section 6, we apply to finite gap Jacobi matrices and so get Theorem 1.3. Section 7 applies the decoupling results of Section 2 to Dirac operators.

\section{Two decoupling lemmas}

We need two basic decoupling facts: one, basically well known, and the second, Theorem 1.4. All our operators act on a separable Hilbert space. The following is essentially a variant of the argument used to prove the Ky Fan inequalities and is stated formally for ease of later use. It is well known.

PROPOSITION 2.1

If $C$ and $D$ are compact self-adjoint operators and $c, d$ are in $(0, \infty)$, then

$$
N(C+D>c+d) \leq N(C>c)+N(D>d) .
$$

\section{Proof}

Let $m=N(C>c), n=N(D>d)$, and let $\varphi_{1}, \ldots, \varphi_{m}$ (resp., $\left.\psi_{1}, \ldots, \psi_{n}\right)$ be a basis for $\operatorname{Ran}\left(P_{(c, \infty)}(C)\right)$ (resp., $\left.\operatorname{Ran}\left(P_{(d, \infty)}(D)\right)\right)$. If $\eta \perp\left\{\varphi_{j}\right\}_{j=1}^{m} \cup\left\{\psi_{j}\right\}_{j=1}^{n}$, then $\langle\eta, C \eta\rangle \leq c$ and $\langle\eta, D \eta\rangle \leq d$. It follows from the min-max principle that $C+D$ has at most $n+m$ eigenvalues above $c+d$. 


\section{COROLLARY 2.2}

If $S, T$ are compact operators and $c, d>0$, then

$$
N\left((S+T)^{*}(S+T)>c+d\right) \leq N\left(S^{*} S>\frac{1}{2} c\right)+N\left(T^{*} T>\frac{1}{2} d\right) .
$$

\section{Proof}

The proof is immediate from (2.1) and

$$
(S+T)^{*}(S+T) \leq(S+T)^{*}(S+T)+(S-T)^{*}(S-T)=2\left(S^{*} S+T^{*} T\right) .
$$

The key to our proof of Theorem 1.4 (which we recall appears in [42]) is the following Proposition 2.3, for which we give a proof involving finite approximation at the end of this section. The appendix has an alternate proof that is more natural to those who know about the relative index of projections (see [3]), but it involves some machinery that is not so commonly known. $\delta_{ \pm}$are defined just before (1.15).

\section{PROPOSITION 2.3}

Let $A$ be a semibounded self-adjoint operator, and let $B_{ \pm}$be two nonnegative relatively $A$-compact factorizable forms. Let $E \notin \sigma(A), \sigma\left(A+B_{+}\right), \sigma\left(A-B_{-}\right), \sigma\left(A+B_{+}-\right.$ $\left.B_{-}\right)$. Then

$$
\delta_{+}\left(A, B_{+} ; E\right)-\delta_{-}\left(A+B_{+}, B_{-} ; E\right)=-\delta_{-}\left(A, B_{-} ; E\right)+\delta_{+}\left(A-B_{-}, B_{+} ; E\right) .
$$

\section{Remark}

This asserts the intuitive fact that the net number of eigenvalues crossing $E$ in going from $A$ to $A+B_{+}-B_{-}$does not depend on the order in which we turn on $B_{+}$and $B_{-}$. It is obvious in the finite-dimensional case, and we will prove it by approximation by finite-dimensional matrices. It allows us to use different orders $A \rightarrow A+B_{+} \rightarrow$ $A+B_{+}-B_{-}$and $A \rightarrow A-B_{-} \rightarrow A+B_{+}-B_{-}$at $\alpha$ and at $\beta$.

\section{Proof of Theorem 1.4}

By (2.4) (with $E=\beta$ ) and (1.17),

$$
\begin{aligned}
N\left(A+B_{+}-B_{-} \in(\alpha, \beta)\right)= & \delta_{+}\left(A, B_{+} ; \alpha\right)-\delta_{-}\left(A+B_{+}, B_{-} ; \alpha\right) \\
& +\delta_{-}\left(A, B_{-} ; \beta\right)-\delta_{+}\left(A-B_{-}, B_{+} ; \beta\right)
\end{aligned}
$$

(1.21) then follows from (1.18) and (1.19) and dropping two negative terms.

We now turn to the proof of Proposition 2.3. 


\section{LEMMA 2.4}

Let $A$ be semibounded and self-adjoint, let $B$ be a relatively $A$-compact, positive, factorizable quadratic form, and let $E \notin \sigma(A), \sigma(A \pm B)$. Then there exist $B_{n}$, positive, finite-rank bounded operators, so that $\delta_{ \pm}\left(A, B_{n} ; E\right)=\delta_{ \pm}(A, B ; E)$ and $B_{n}^{1 / 2}(A-E)^{-1} B_{n}^{1 / 2}$ converge in norm to $B^{1 / 2}(A-E)^{-1} B^{1 / 2}$.

\section{Proof}

By (1.18) and (1.19), it suffices to prove the norm convergence. Let $\mathscr{H}_{ \pm 1}$ be the scale associated to $A$ (see [44]). Let $B: \mathscr{H}_{-1} \rightarrow \mathscr{H}_{+1}$ with $B=C^{*} C$. Note that $C$ is compact, so it can be approximated by finite rank operators with vectors in $\mathscr{H}$ and $\mathcal{K}$.

\section{LEMMA 2.5}

Let $A$ be a semibounded operator with $E \notin \sigma(A)$ and $F \subset \mathscr{H}$ a finite-dimensional space. Then there exist $A_{n}$, finite-rank operators, with $F \subset \operatorname{Ran}\left(A_{n}-E Q_{n}\right)$ (where $Q_{n}$ is the projection onto $\left.\operatorname{Ran}\left(A_{n}\right)\right)$, so that $B^{1 / 2}\left(A_{n}-E Q_{n}\right)^{-1} B^{1 / 2} \rightarrow B^{1 / 2}(A-E)^{-1} B^{1 / 2}$ in norm as $n \rightarrow \infty$ for all finite-rank, nonnegative $B$ with $\operatorname{Ran}(B) \subset F$.

\section{Proof}

Define $f_{n}(x): \mathbb{R} \rightarrow \mathbb{R}$ by

$$
f_{n}(x)= \begin{cases}-n & \text { if } x \leq-n, \\ n & \text { if } x \geq n, \\ \frac{1}{n}[n x] & \text { if }-n \leq x \leq n,\end{cases}
$$

where $[y]=$ integral part of $y$. Let $\tilde{A}_{n}=f_{n}(A)$; so $\left\|\left(\tilde{A}_{n}-E\right)^{-1}-(A-E)^{-1}\right\| \rightarrow 0$. Let $Q_{n}$ be the projection onto the cyclic subspace generated by $\tilde{A}_{n}$ and $F$. This cyclic subspace is finite-dimensional, so $A_{n}=Q_{n} \tilde{A}_{n} Q_{n}$ is of finite rank, and if $\operatorname{Ran}(B) \subset F$, $B^{1 / 2}\left(\tilde{A}_{n}-E\right)^{-1} B^{1 / 2}=B^{1 / 2}\left(A_{n}-E Q_{n}\right)^{-1} B^{1 / 2}$.

\section{Proof of Proposition 2.3}

If $A, B_{+}$, and $B_{-}$are operators on a finite-dimensional space, then (2.4) is immediate since both sides equal $\operatorname{dim}\left[\operatorname{Ran}\left(P_{(-\infty, E)}(A)\right)\right]-\operatorname{dim}\left[\operatorname{Ran}\left(P_{(-\infty, E)}\left(A+B_{+}-B_{-}\right)\right)\right]$. By Lemmas 2.4 and 2.5, we can find finite-dimensional $A_{n}$ and $\left(B_{n}\right)_{ \pm}$so that all $\delta$ objects in (2.4) equal the $A, B_{ \pm}$objects.

\section{CLR bounds for regular gaps in periodic Schrödinger operators}

Let $V_{0}$ be a periodic, locally $L^{v / 2}$-function on $\mathbb{R}^{v}$ for $v \geq 3$; that is,

$$
V_{0}\left(x+\tau_{j}\right)=V_{0}(x)
$$


for $\tau_{1}, \ldots, \tau_{v}$ linearly independent in $\mathbb{R}^{v}$. Let $H_{0}=-\Delta+V_{0}$. Then $H_{0}$ is a direct integral of operators, $H_{0}(k)$, with compact resolvent, where $k$ runs through a fundamental cell of the dual lattice (see, e.g., [43]). Let $\varepsilon_{1}(k) \leq \varepsilon_{2}(k) \leq \cdots$ be the eigenvalues of $H_{0}(k)$. Let $(\alpha, \beta)$ be a gap in $\sigma\left(H_{0}\right)$ in that $(\alpha, \beta) \cap \sigma\left(H_{0}\right)=\emptyset$ but $\alpha, \beta \in \sigma\left(H_{0}\right)$. We say $\beta$ (resp., $\alpha$ ) is a regular band edge if and only if

(i) $\quad \beta=\inf _{k} \varepsilon_{n}(k)\left(\right.$ resp., $\left.\alpha=\sup _{k} \varepsilon_{n}(k)\right)$ for a single $n$;

(ii) $\quad \varepsilon_{n}(k)=\beta\left(\right.$ resp., $\left.\varepsilon_{n}(k)=\alpha\right)$ has finitely many solutions $k^{(1)}, \ldots, k^{(\ell)}$;

(iii) at each $k^{(j)}, \varepsilon_{n}(k)$ has a matrix of second derivatives which is strictly positive (resp., strictly negative).

We say that $(\alpha, \beta)$ is a regular gap if both band edges are regular. It is believed that for a generic $V_{0}$, all band edges are regular (for generic results on (i), (ii), see Klopp and Ralston [36]). Birman [9] has proved that if $(\alpha, \beta)$ is a regular gap, then with $\|\cdot\|_{\mathcal{\alpha}_{v / 2}^{w}}$ the weak trace class norm (see [48]), one has a constant $c$ so that

$$
\sup _{\lambda \in(\alpha, \beta)}\left\||W|^{1 / 2}\left(H_{0}-\lambda\right)^{-1}|W|^{1 / 2}\right\|_{q_{v / 2}^{w}} \leq c\|W\|_{v / 2} .
$$

By combining this with Theorem 1.4, one immediately has the following.

THEOREM 3.1

If $(\alpha, \beta)$ is a regular gap of $H_{0}$, then for any $W \in L^{v / 2}\left(\mathbb{R}^{v}\right)$, we have

$$
N\left(H_{0}+W \in(\alpha, \beta)\right) \leq c \int_{\mathbb{R}^{v}}|W(x)|^{v / 2} d^{v} x .
$$

Because he did not have Theorem 1.4, Birman restricted himself to perturbations of a definite sign.

Obviously, if there are finitely many gaps, one can sum over all gaps if they are all regular. It is known (see Sobolev [52] and references therein) that if $V_{0}$ is smooth, then there are always only finitely many gaps.

\section{An abstract critical Lieb-Thirring bound}

In this section, we prove the following continuum critical Lieb-Thirring bound and discrete analogue.

\section{THEOREM 4.1}

Let $H_{0}$ be a semibounded self-adjoint operator on $L^{2}(\mathbb{R}, d x)$ so that for some $a<b$, the following hold.

(i) We have

$$
[a, b) \cap \sigma\left(H_{0}\right)=\emptyset .
$$

(ii) For $E_{0}<\inf \sigma\left(H_{0}\right),\left(H_{0}-E_{0}\right)^{-1 / 2}$ is a bounded operator from $L^{2}$ to $L^{\infty}$. 
(iii) There exist $\varepsilon, \delta>0$ and continuous functions $\rho, \theta, E$ from $(-\delta, \delta)$ to $\mathbb{R}$ and $u(\cdot, \cdot)$ from $\mathbb{R} \times(-\delta, \delta)$ to $\mathbb{C}$, so that any $\varphi \in \operatorname{Ran}\left(P_{[b, b+\varepsilon)}\left(H_{0}\right)\right)$ has an expansion

$$
\varphi(x)=\int_{-\delta}^{\delta} \widetilde{\varphi}(k) u(x, k) d k
$$

with

$$
\widetilde{H_{0}} \varphi(k)=E(k) \widetilde{\varphi}(k)
$$

and

$$
\|\varphi\|_{L^{2}(\mathbb{R}, d x)}^{2}=\int|\widetilde{\varphi}(k)|^{2} \rho(k) d k .
$$

Moreover, for any $\widetilde{\varphi} \in L^{2}(-\delta, \delta ; d k)$, (4.2) defines a function in $L^{2}(\mathbb{R})$ lying in $\operatorname{Ran}\left(P_{[b, b+\varepsilon)}\left(H_{0}\right)\right)$. (The integral converges by the hypothesis (4.7) below.)

(iv) We have

$$
0<\inf _{k \in(-\delta, \delta)} \rho(k)=\rho_{-} \leq \sup _{k \in(-\delta, \delta)} \rho(k)=\rho_{+}<\infty
$$

(v) $\quad E(k)=E(-k)$ and maps $[0, \delta)$ bijectively onto $[0, \varepsilon)$. For some $c_{1}>0$, we have

$$
E(k) \geq b+c_{1} k^{2} .
$$

(vi) We have

$$
\sup _{\substack{k \in(-\delta, \delta) \\ x \in \mathbb{R}}}|u(x, k)|=c_{2}<\infty .
$$

(vii) If

$$
v(x, k)=e^{-i \theta(k) x} u(x, k),
$$

then for some $c_{3}<\infty$ and all $x \in \mathbb{R}$,

$$
|v(x, k)-v(x, 0)| \leq c_{3} k^{2} .
$$

(viii) $\theta$ is $C^{2}$ on $(-\delta, \delta)$ and

$$
\inf _{k \in(-\delta, \delta)} \theta^{\prime}(k)>0 .
$$


(ix) We have

$$
\begin{aligned}
& E(-k)=E(k), \quad u(x,-k)=\overline{u(x, k)}, \quad \theta(-k)=-\theta(k), \\
& \rho(-k)=\rho(k) .
\end{aligned}
$$

Then for some $C$ and all $V \in L^{1}(\mathbb{R}, d x)$, we have

$$
\sum_{\substack{e \in \sigma_{\mathrm{d}}\left(H_{0}+V\right) \\ e \in(a, b)}}(b-e)^{1 / 2} \leq C \int|V(x)| d x .
$$

\section{Remarks}

(1) There is a similar result for $(b, a] \cap \sigma\left(H_{0}\right)=\emptyset$ with (4.6) replaced by

$$
E(k) \leq b-c_{1} k^{2}
$$

This means that we can control full gaps $\left(b_{-}, b_{+}\right)$in $\sigma\left(H_{0}\right)$. To control $\left(-\infty\right.$, inf $\left.\sigma\left(H_{0}\right)\right)$ (and the top half in the discrete case) requires an additional argument that we provide at the end of this section.

(2) We could replace $\theta(k)$ by $k$ (and we will essentially do that). We have not because, in the finite gap case, there is a natural parameter distinct from $\theta$.

(3) The idea behind the proof is to use decoupling to reduce the proof to control of the $[b, b+\varepsilon)$-region and use the eigenfunction expansion there to compare to $-\frac{d^{2}}{d x^{2}}+\widetilde{V}(x)$, where $\widetilde{V}$ and $V$ have comparable $L^{1}$-norms.

(4) Hypothesis (ii) implies that any $V \in L^{1}$ is a relatively compact perturbation of $H_{0}$.

(5) The decomposition we use in the proof below was suggested to us by a paper of Sobolev [51], who used it in a related, albeit distinct, context.

(6) Equations (4.2) and (4.4) imply that for all $\tilde{\varphi} \in L^{2}((-\delta, \delta), d k)$ and all $\psi \in$ $\operatorname{Ran}\left(P_{[b, b+\varepsilon)}\left(H_{0}\right)\right)$, we have

$$
\begin{aligned}
\langle\psi, \varphi\rangle & =\int_{-\delta}^{\delta} d k \int d x \widetilde{\varphi}(k) \overline{\psi(x)} u(x, k) \\
& =\int \bar{\psi}(k) \widetilde{\varphi}(k) \rho(k) d k,
\end{aligned}
$$

which implies that

$$
\widetilde{\psi}(k)=\rho(k)^{-1} \int d x \overline{u(x, k)} \psi(x) .
$$

We will prove (4.12) by reducing it to a bound on $N\left(H_{0}+V \in[a, b-\tau]\right)$. 


\section{LEMMA 4.2}

If we have $C_{1}, C_{2}, C_{3}$ such that, for almost every $0<\tau<b-a$,

$$
N\left(H_{0}+V \in[a, b-\tau]\right) \leq C_{1} \int|V(x)| d x+N\left(-\frac{d^{2}}{d x^{2}}-C_{2} V_{-} \leq-\frac{\tau}{C_{3}}\right),
$$

then (4.12) holds.

\section{Remark}

For control of a lower band edge, $V_{-}$in the last term is replaced by $V_{+}$.

Proof

For any absolutely continuous function, $f$, on $[a, b]$ with $f(b)=0$,

$$
\sum_{\substack{e \in \sigma_{\mathrm{d}}\left(H_{0}+V\right) \\ e \in[a, b]}} f(e)=-\int_{0}^{b-a} f^{\prime}(b-\tau) N\left(H_{0}+V \in[a, b-\tau]\right) d \tau,
$$

so by (4.15) with $f(y)=(b-y)^{1 / 2}$,

\section{LHS of (4.12)}

$$
\begin{aligned}
& \leq \int_{0}^{b-a} \frac{1}{2} \tau^{-1 / 2}\left[C_{1}\|V\|_{1}+N\left(-\frac{d^{2}}{d x^{2}}-C_{2} V_{-} \leq-\frac{\tau}{C_{3}}\right)\right] d \tau \\
& =(\sqrt{b-a}) C_{1}\|V\|_{1}+\sqrt{C_{3}} \int_{0}^{(b-a) / C_{3}} \frac{1}{2} \sigma^{-1 / 2} N\left(-\frac{d^{2}}{d x^{2}}-C_{2} V_{-} \leq-\sigma\right) d \sigma \\
& \leq(\sqrt{b-a}) C_{1}\left\|V_{1}\right\|+\sqrt{C_{3}} \sum_{e<0}(-e)^{1 / 2} \\
& \leq\left(\sqrt{b-a} C_{1}+C_{2} \sqrt{C_{3}} L_{\frac{1}{2}, 1}\right)\|V\|_{1},
\end{aligned}
$$

proving (4.12). (It is known that $L_{1 / 2,1}=1 / 2$ [31].)

\section{LEMMA 4.3}

Suppose $E_{0}<\inf \sigma\left(H_{0}\right)$ and $\left(H_{0}-E_{0}\right)^{-1 / 2}$ is a bounded operator from $L^{2}$ to $L^{\infty}$. Let $f(x)$ be a function on $\sigma\left(H_{0}\right)$ with

$$
D=\sup _{y \in \sigma\left(H_{0}\right)}|f(y)|\left(y-E_{0}\right)<\infty
$$

Then for any $V \in L^{1},|V|^{1 / 2} f\left(H_{0}\right)|V|^{1 / 2}$ is trace class and

$$
\left\||V|^{1 / 2} f\left(H_{0}\right)|V|^{1 / 2}\right\|_{1} \leq D\left\|\left(H-E_{0}\right)^{-1 / 2}\right\|_{2, \infty}^{2}\|V\|_{1}
$$

(where the $\|\cdot\|_{1}$ on the left is trace class norm and on the right is $L^{1}(\mathbb{R})$-norm). 


\section{Proof}

By the Dunford-Pettis theorem (see [53]), $\left(H_{0}-E_{0}\right)^{-1 / 2}$ has a Hermitian symmetric integral kernel $K(x, y)$ with

$$
\sup _{x}\left(\int|K(x, y)|^{2} d y\right)^{1 / 2}=\left\|\left(H-E_{0}\right)^{-1 / 2}\right\|_{2, \infty},
$$

so, by the symmetry, $\left(H-E_{0}\right)^{-1 / 2}|V|^{1 / 2}$ is Hilbert-Schmidt with Hilbert-Schmidt norm bounded by $\left\|\left(H-E_{0}\right)^{-1 / 2}\right\|_{2, \infty}\|V\|_{1}^{1 / 2}$. Since $D$ is the operator norm of $\left(H_{0}-\right.$ $\left.E_{0}\right) f\left(H_{0}\right),(4.18)$ is immediate.

\section{Proof of Theorem 4.1}

We use (1.21) with $A=H_{0}, B=V, \alpha=a, \beta=b-\tau$, where $\tau$ is any point in $(0, b-a)$, and Lemma 4.3 to see

$$
\text { LHS of }(4.15) \leq N\left(V_{-}^{1 / 2}\left(H_{0}-b+\tau\right)^{-1} V_{-}^{1 / 2}>1\right)+C \int\left|V_{+}(x)\right| d x
$$

for a suitable constant.

In the first term of (4.19), we insert $P_{[b, b+\varepsilon]}\left(H_{0}\right)+\left(1-P_{[b, b+\varepsilon]}\left(H_{0}\right)\right)$ in $\left(H_{0}-\right.$ $b+\tau)^{-1}$, use (2.1) with $c=d=1 / 2$, and use Lemma 4.3 to get

$$
\begin{aligned}
& N\left(V_{-}^{1 / 2}\left(H_{0}-b+\tau\right)^{-1} V_{-}^{1 / 2}>1\right) \\
& \quad \leq C \int\left|V_{-}(x)\right| d x+N\left(V_{-}^{1 / 2}\left(H_{0}-b+\tau\right)^{-1} P_{[b, b+\varepsilon]}\left(H_{0}\right) V_{-}^{1 / 2}>\frac{1}{2}\right) .
\end{aligned}
$$

By (4.2) - (4.4) and (4.14), for $\lambda \equiv b-\tau \notin \sigma\left(H_{0}\right),\left(H_{0}-\lambda\right)^{-1} P_{[b, b+\varepsilon)}\left(H_{0}\right)$ has the integral kernel

$$
\int_{-\delta}^{\delta} \frac{u(x, k) \overline{u(y, k)}}{E(k)-b+\tau} \frac{d k}{\rho(k)} .
$$

Write

$$
u(x, k)=e^{i \theta(k) x} v(x, 0)+e^{i \theta(k) x}[v(x, k)-v(x, 0)],
$$

and insert into (4.21), writing the kernel as $\left(S_{\tau}+T_{\tau}\right)^{*}\left(S_{\tau}+T_{\tau}\right)$, and use (2.2), where $S, T$ have integral kernels

$$
S_{\tau}(k, x)=(E(k)-b+\tau)^{-1 / 2} \rho(k)^{-1 / 2} e^{i \theta(k) x} v(x, 0),
$$

and similarly for $T$. 
By (4.5), (4.6), and (4.9), uniformly in $k, x$, and $\tau,\left|T_{\tau}(k, x)\right|$ is bounded, so $T_{\tau} V_{-}^{1 / 2}$ is bounded uniformly in $\tau$ in the Hilbert-Schmidt norm as a map from $L^{2}(\mathbb{R}, d x)$ to $L^{2}([b, b+\varepsilon), d k)$. Thus, uniformly in $\tau$,

$$
N\left(V_{-}^{1 / 2} T_{\tau}^{*} T_{\tau} V_{-}^{1 / 2}>\frac{1}{8}\right) \leq C \int\left|V_{-}(x)\right| d x .
$$

Let $Q(\theta)$ be an inverse function to $\theta$. Changing variables from $k$ to $\theta, S_{\tau}^{*} S_{\tau}$ has integral kernel

$$
\int_{-\theta(\delta)}^{\theta(\delta)} \frac{v(x, 0) \overline{v(y, 0)} e^{i \theta(x-y)}}{E(Q(\theta))-b+\tau} \frac{d \theta}{\theta^{\prime}(Q(\theta)) \rho(Q(\theta))} .
$$

By (4.10) and (4.6), there is a constant $c_{4}$ with $E(Q(\theta))-b+\tau \geq c_{4} \theta^{2}+\tau$. Also, $u \bar{u}$ is a positive definite kernel, so the operator in (4.25) is dominated in the operator sense by the kernel

$$
c_{5} \int_{-\infty}^{\infty} \frac{v(x, 0) \overline{v(y, 0)} e^{i \theta(x-y)}}{c_{4} \theta^{2}+\tau} d \theta,
$$

which is the integral kernel of $c_{5} v(\cdot, 0)\left(-c_{4} \frac{d^{2}}{d x^{2}}+\tau\right)^{-1} \overline{v(\cdot, 0)}$. Thus,

$$
\begin{aligned}
& N\left(V_{-}^{1 / 2} S_{\tau}^{*} S_{\tau} V_{-}^{1 / 2}>\frac{1}{8}\right) \\
& \quad=N\left(8 c_{5} v(\cdot, 0) V_{-}^{1 / 2}\left(-c_{4} \frac{d^{2}}{d x^{2}}+\tau\right)^{-1} v(\cdot, 0) V_{-}^{1 / 2}>1\right) \\
& \quad=N\left(-\frac{d^{2}}{d x^{2}}-\frac{8 c_{5}}{c_{4}}|v(\cdot, 0)|^{2} V_{-}<-\frac{\tau}{c_{3}}\right)
\end{aligned}
$$

by the Birman-Schwinger principle.

Letting $C_{2}=\frac{8 c_{5}}{c_{4}} \sup _{x}|v(\cdot, 0)|^{2}$, we see that (4.15), and so (4.12), holds.

Next, we turn to the analogue for Jacobi matrices. $J_{0}$ is a fixed two-sided Jacobi matrix, and $\delta J_{0}$ is a Jacobi perturbation with parameters $\left\{a_{n}^{(0)}, b_{n}^{(0)}\right\}_{n=-\infty}^{\infty}$ and $\left\{\delta a_{n}, \delta b_{n}\right\}_{n=-\infty}^{\infty}$, respectively. We have $J=J_{0}+\delta J$ with parameters $\left\{a_{n}, b_{n}\right\}_{n=-\infty}^{\infty}$.

THEOREM 4.4

Let $J_{0}$ be a Jacobi matrix on $\ell^{2}(\mathbb{Z})$, so that for some $a<b$, the following hold.

(i) We have

$$
[a, b) \cap \sigma\left(J_{0}\right)=\emptyset .
$$


(ii) There exist $\varepsilon, \delta>0$ and functions $\rho, \theta, E$ from $(-\delta, \delta)$ to $\mathbb{R}$ and $u$.(.) from $\mathbb{Z} \times(-\delta, \delta)$ to $\mathbb{C}$ so that any $\varphi \in \operatorname{Ran}\left(P_{[b, b+\varepsilon)}\left(J_{0}\right)\right)$ has an expansion

$$
\varphi_{n}=\int_{-\delta}^{\delta} \widetilde{\varphi}(k) u_{n}(k) d k
$$

with

$$
\widetilde{J_{0} \varphi}(k)=E(k) \widetilde{\varphi}(k)
$$

and

$$
\|\varphi\|_{\ell^{2}(\mathbb{Z})}^{2}=\int|\widetilde{\varphi}(k)|^{2} \rho(k) d k .
$$

Moreover, for any $\widetilde{\varphi} \in L^{2}((-\delta, \delta), d k)$, (4.30) defines a $\varphi \in$ $\operatorname{Ran}\left(P_{[b, b+\varepsilon)}\left(J_{0}\right)\right)$.

(iii) We have

$$
0<\inf _{k \in(-\delta, \delta)} \rho(k)=\rho_{-} \leq \sup _{k \in(-\delta, \delta)} \rho(k)=\rho_{+}<\infty
$$

(iv) We have $E(k)=E(-k)$ and maps $[0, \delta)$ to $[0, \varepsilon)$. For some $c_{1}>0$, we have

$$
E(k) \geq b+c_{1} k^{2}
$$

(v) We have

$$
\sup _{\substack{k \in(-\delta, \delta) \\ n \in \mathbb{Z}}}\left|u_{n}(k)\right|=c_{2}<\infty .
$$

(vi) If

$$
v_{n}(k)=e^{-i \theta(k) n} u_{n}(k),
$$

then for some $c_{3}<\infty$ and all $n \in \mathbb{Z}$,

$$
\left|v_{n}(k)-v_{n}(0)\right| \leq c_{3} k^{2}
$$

(vii) $\theta$ is $C^{2}$ on $(-\delta, \delta)$ and

$$
\inf _{x \in(-\delta, \delta)} \theta^{\prime}(k)>0
$$


(viii) We have

$$
\begin{aligned}
& E(-k)=E(k), \quad u_{n}(-k)=\overline{u_{n}(k)}, \quad \theta(-k)=-\theta(k), \\
& \rho(-k)=\rho(k) .
\end{aligned}
$$

Then for some $C$ and all $\delta J$, we have

$$
\sum_{\substack{e \in \sigma_{\mathrm{d}}\left(J_{0}+\delta J\right) \\ e \in(a, b)}}(b-e)^{1 / 2} \leq C \sum_{n=-\infty}^{\infty}\left|\delta a_{n}\right|+\left|\delta b_{n}\right| .
$$

The analogue of $\left(H_{0}-E\right)^{-1 / 2}$ bounded from $L^{2}$ to $L^{\infty}$ is missing since $\ell^{2} \subset \ell^{\infty}$, and thus,

$$
\left\|\left(J_{0}-E_{0}\right)^{-1 / 2} f\right\|_{\infty} \leq \operatorname{dist}\left(E_{0}, \sigma\left(J_{0}\right)\right)^{-1 / 2}\|f\|_{2}
$$

With this remark and the bound of [32], the proof is identical to that of Theorem 4.1 if we use an additional argument. Following [32], we define $\delta J_{ \pm}$to be the Jacobi matrices with parameters

$$
\begin{aligned}
& \delta b_{n}^{ \pm}=\max \left\{0, \pm b_{n}\right\}+\frac{1}{2} a_{n}+\frac{1}{2} a_{n+1}, \\
& \delta a_{n}^{ \pm}= \pm \frac{1}{2} a_{n},
\end{aligned}
$$

so $\delta J_{ \pm} \geq 0$ as matrices, $\delta J=\delta J_{+}-\delta J_{-}$, and

$$
\left\|\left(\delta J_{ \pm}\right)^{1 / 2}\right\|_{\mathrm{HS}}^{2}=\operatorname{Tr}\left(\delta J_{ \pm}\right) \leq \sum_{n}\left|b_{n}\right|+2 a_{n} .
$$

Finally, we need to say something about the sum over eigenvalues on semiinfinite intervals but a distance 1 from $\sigma\left(H_{0}\right)$ or $\sigma\left(J_{0}\right)$ (since Theorems 4.1 and 4.4 control the sum of (inf $\sigma\left(J_{0}\right)-1, \inf \sigma\left(H_{0}\right)$ ), and similarly for $\left.J_{0}\right)$. We discuss the discrete case first.

PROPOSITION 4.5

Let $A$ be a bounded operator on a Hilbert space, and let $B$ be trace class with $\alpha=\inf \sigma(A)$. Then

$$
\sum_{\substack{e \in \sigma_{\mathrm{d}}(A+B) \\ e \leq \alpha-1}}(\alpha-e)^{1 / 2} \leq \operatorname{Tr}(|B|)
$$




\section{Proof}

Let $\left\{e_{n}\right\}_{n=1}^{\infty}$ be a counting of the eigenvalues in $(-\infty, \alpha-1)$, and let $\left\{\varphi_{n}\right\}_{n=1}^{\infty}$ be the eigenvectors. Then, since $\alpha-e_{n} \geq 1$,

$$
\begin{aligned}
\sum_{n=1}^{\infty}\left(\alpha-e_{n}\right)^{1 / 2} & \leq \sum_{n=1}^{\infty}\left(\alpha-e_{n}\right) \\
& \leq \sum_{n=1}^{\infty}\left(\varphi_{n},(\alpha-A) \varphi_{n}\right)-\left(\varphi_{n}, B \varphi_{n}\right) \\
& \leq \sum_{n=1}^{\infty}\left(\varphi_{n}, B_{-} \varphi_{n}\right) \\
& \leq \operatorname{Tr}(|B|)
\end{aligned}
$$

where (4.45) comes from $A \geq \alpha$.

PROPOSITION 4.6

Let $h_{0}=-\frac{d^{2}}{d x^{2}}$ on $L^{2}(\mathbb{R}, d x)$. Let $H_{0}$ be an operator for which, for some $\gamma>0$,

$$
H_{0} \geq \gamma h_{0}+\beta \text {. }
$$

Let $\alpha=\inf \sigma\left(H_{0}\right)$. Then there exist $C_{1}, C_{2}>0$, so that for all $V \in L^{1}$,

$$
\sum_{\substack{e \in \sigma_{\mathrm{d}}\left(H_{0}+V\right) \\ e<\alpha-C_{1}}}(\alpha-e)^{1 / 2} \leq C_{2} \int|V(x)| d x .
$$

\section{Proof}

By (4.47), $\beta \leq \alpha$. Let $e<\beta$. Then, by (4.47),

$$
\begin{aligned}
N\left(H_{0}+V \leq e\right) & \leq N\left(\gamma h_{0}+V \leq e-\beta\right) \\
& =N\left(h_{0}+\gamma^{-1} V \leq \gamma^{-1}(e-\beta)\right),
\end{aligned}
$$

so using the critical Lieb-Thirring bound for $h_{0}$,

$$
\sum_{e<\beta} \sqrt{\gamma^{-1}(\beta-e)} \leq \frac{1}{2} \gamma^{-1} \int|V(x)| d x .
$$

If $e<\beta-1$, then $\alpha-e \leq(\beta-e)(\alpha-\beta+1)$, so

$$
\sum_{e<\beta-1} \sqrt{\alpha-e} \leq \frac{1}{2}(\alpha-\beta+1)^{1 / 2} \gamma^{-1 / 2} \int|V(x)| d x .
$$




\section{One-dimensional periodic Schrödinger operators}

In this section, we prove Theorem 1.1; that is, we prove critical Lieb-Thirring bounds in individual gaps for perturbations of periodic Schrödinger operators. So $h_{0}=-\frac{d^{2}}{d x^{2}}$ on $L^{2}(\mathbb{R}, d x)$, and $V_{0}$ is a periodic potential with

$$
V_{0}(x+2 \pi)=V_{0}(x) .
$$

(There is no loss with picking the period to be $2 \pi$.) We suppose

$$
\int_{-\pi}^{\pi}\left|V_{0}(x)\right| d x<\infty .
$$

Then, by a Sobolev estimate, $V_{0}$ is a form-bounded perturbation of $h_{0}$ with relative bound zero. Thus, $H_{0}=h_{0}+V_{0}$ is a well-defined form sum, and if $E_{0}<\inf \sigma\left(H_{0}\right)$, then $\left(h_{0}+1\right)^{1 / 2}\left(H_{0}-E_{0}\right)^{-1 / 2}$ is bounded from $L^{2}$ to $L^{2}$. So by a Sobolev estimate, $\left(H_{0}-E_{0}\right)^{-1 / 2}$ is bounded from $L^{2}$ to $L^{\infty}$; that is, Theorem 4.1(ii) is valid.

The following facts are well known (see [43, Section XIII.16], which supposes $V_{0}$ bounded, but no changes are needed to handle the locally $L^{1}$-case; see also [40]):

(i) If $U: L^{2}(\mathbb{R}, d x) \rightarrow L^{2}\left([0,2 \pi), L^{2}([0,2 \pi], d x) ; \frac{d \varphi}{2 \pi}\right)$ is defined by

$$
(U f)_{\varphi}(x)=\sum_{n=-\infty}^{\infty} e^{-i \varphi n} f(x+2 \pi n),
$$

then $U$ is unitary.

(ii) If $h_{0}(\varphi)$ is defined for $\varphi \in[0,2 \pi)$ on $L^{2}([0,2 \pi], d x)$ as $-\frac{d^{2}}{d x^{2}}$ with boundary conditions

$$
u(2 \pi)=e^{i \varphi} u(0), \quad u^{\prime}(2 \pi)=e^{i \varphi} u^{\prime}(0)
$$

and $H(\varphi)=h_{0}(\varphi)+V_{0}$, then

$$
U H U^{-1} g_{\varphi}=H(\varphi) g_{\varphi} .
$$

(iii) Each $H(\varphi)$ has compact resolvent and has eigenvalues $\left\{\varepsilon_{j}(\varphi)\right\}_{j=1}^{\infty}$ and eigenvectors $u_{j}^{(\varphi)}(x)$, so that

$$
H(\varphi) u_{j}^{(\varphi)}=\varepsilon_{j}^{(\varphi)} u_{j}^{(\varphi)}
$$

If, for $x \in[0,2 \pi)$,

$$
v_{j}^{(\varphi)}(x)=e^{-i \varphi x / 2 \pi} u_{j}^{(\varphi)}(x)
$$


then by (5.4), $v_{j}$ has a periodic extension and all $v_{j}^{(\varphi)}$ lie in $Q\left(h_{0}(\varphi \equiv 0)\right)$ and obey (where $p=-i d / d x$ )

$$
\left[h_{0}(0)+2 \frac{\varphi}{2 \pi} p+\left(\frac{\varphi}{2 \pi}\right)^{2}+V_{0}\right] v_{j}^{(\varphi)}=\varepsilon_{j}(\varphi) v_{j}^{(\varphi)} .
$$

If the operator in [...] in (5.8) is $\widetilde{H}(\varphi)$, then it is a Kato analytic family of type (B). Moreover, for any single $j, v_{j} \in Q\left(h_{0}\right)$ with bounded norm, by a Sobolev estimate,

$$
\sup _{\varphi, x}\left|v_{j}^{(\varphi)}(x)\right|<\infty
$$

for each fixed $j$.

(iv) We have $\varepsilon_{j}(2 \pi-\varphi)=\varepsilon_{j}(\varphi)$ and $v_{j}^{(2 \pi-\varphi)}=\overline{v_{j}^{(\varphi)}}$. On $[0, \pi],(-1)^{j+1} \varepsilon_{j}$ is strictly monotone increasing, so $\varepsilon_{1}(0)<\varepsilon_{1}(\pi) \leq \varepsilon_{2}(\pi)<\varepsilon_{2}(0) \leq \varepsilon_{3}(0) \leq$ $\cdots<\varepsilon_{2 j-1}(\pi) \leq \varepsilon_{2 j}(\pi)<\varepsilon_{2 j}(0) \leq \varepsilon_{2 j+1}(0) \cdots$. The gaps in $\operatorname{spec}(H)$ are exactly the nonempty $\left(\varepsilon_{2 j-1}(\pi), \varepsilon_{2 j}(\pi)\right)$ and $\left(\varepsilon_{2 j}(0), \varepsilon_{2 j+1}(0)\right)$. If such a gap is nonempty, we say it is an open gap.

(v) There is an entire analytic function $\Delta(E)$ such that

$$
\Delta\left(\varepsilon_{j}(\varphi)\right)=2 \cos (\varphi)
$$

and a gap is open if and only if $\Delta^{\prime}(\varepsilon) \neq 0$ at the endpoints of the gap. It then follows from (5.10) that at an open gap,

$$
\varepsilon_{j}^{\prime}(0 \text { or } \pi)=0, \quad \varepsilon_{j}^{\prime \prime}(0 \text { or } \pi) \neq 0 .
$$

This says that the framework of Theorem 4.1 is applicable. For notational simplicity, we consider an open gap at $\varphi=0$ (below, if $\varphi=\pi$, replace $k=\varphi / 2 \pi$ by $k=$ $(\varphi-\pi) / 2 \pi$; and the associated $v_{j}$ is then antiperiodic) and the top end of the gap at energy $b=\varepsilon_{n}(0)$. We take $\delta=1 / 4, k=\varphi / 2 \pi$, and $\theta(k)=k$. $E(k)=\varepsilon_{n}(2 \pi k)$. For $0 \leq x<2 \pi$,

$$
u(x+2 \pi m, k)=u_{n}^{(2 \pi k)}(x) e^{2 \pi i m k}
$$

by using the boundary condition (5.4). We set $\varepsilon=E(1 / 4)=\varepsilon_{n}(\pi / 2)$. $\operatorname{Ran}\left(P_{[b, b+\varepsilon]}\left(H_{0}\right)\right)$ is exactly those $f$ with $(U f)_{\varphi}=0$ if $\varphi \notin(-\pi / 2, \pi / 2)$ and is equal to a multiple of $u_{n}^{(\varphi)}$ if $\varphi \in(-\pi / 2, \pi / 2)$ :

$$
\tilde{f}(k)=\left\langle(U f)_{(\varphi=2 \pi k)}, u_{n}^{(2 \pi k)}\right\rangle .
$$

Equation (4.4) holds with $\rho(k) \equiv 1$, so (4.5) is immediate; (4.6) holds by the fact that $\varepsilon$ is real analytic on $(-\pi, \pi)$ and (5.11) holds; (4.7) holds by (5.9). 
Equation (4.9) holds because $v$ is periodic in $x$ and $u$ is real analytic in $k$ with $\frac{d u}{d k}=0$; (viii) and (ix) of Theorem 4.1 are immediate.

Theorem 4.1 thus implies Theorem 1.1.

We have only controlled individual gaps. It is natural to ask if one can sum over all the typically infinitely many gaps. We believe this will be difficult with our methods. The issue involves the constant $c_{3}$ in (4.9). For large $n$, the $n$th band has size $O(n)$ near an energy of $O\left(n^{2}\right)$. The size $g_{n}$ of the $n$th gap is small. If $v_{0}$ is $C^{\infty}$, it is known (see Hochstadt [29]) that $g_{n}=o\left(n^{k}\right)$ for all $k$; and for $v_{0}(x)=\lambda \cos (x)$, it is known (see [4]) that $g_{n} \sim n^{-2 n}$. Away from $k=0$ or $\pi, \varepsilon_{n}^{\prime}(k) \sim n$, and it goes from $\varepsilon_{n}^{\prime}=0$ to $n$ in a distance of size $O\left(g_{n}\right)$; that is, we expect $\varepsilon_{n}^{\prime \prime}(0) \sim g_{n}^{-1} n$. Thus, we expect $c_{3}$ to be $O\left(n g_{n}^{-1}\right)$. While $c_{3}$ is divided by $c_{1}$, which is also large, $c_{3} \sim \sup _{|k| \leq \delta} \varepsilon_{n}^{\prime \prime}(k)$, while $c_{1} \sim \inf _{|k| \leq \delta} \varepsilon^{\prime \prime}(k)$. So unless we take $\delta \downarrow 0$ (which itself causes difficulties), the cancellation is only be partial. Thus, we have not been able to sum over all gaps.

\section{Critical Lieb-Thirring bounds and generalized Nevai conjecture for finite gap Jacobi matrices}

In this section, we turn to perturbations of elements of the isospectral torus of Jacobi matrices assigned to a finite gap set, $\mathfrak{e}$, as described in the introduction. Our main goal is the following.

\section{THEOREM 6.1}

Let $\mathfrak{e}$ be a finite gap set, and let $\left(\beta_{j}, \alpha_{j+1}\right)$ be a gap in $\mathbb{R} \backslash \mathfrak{e}$. Let $\left\{a_{n}^{(0)}, b_{n}^{(0)}\right\}_{n=-\infty}^{\infty}$ be an element of the isospectral torus. Then for a constant $C$ and any $\left\{a_{n}, b_{n}\right\}_{n=1}^{\infty}$ a set of Jacobi parameters obeying the two-sided analogue of (1.11),

$$
\sum_{e \in\left(\beta_{j}, \alpha_{j+1}\right) \cap \sigma(J)} \operatorname{dist}\left(e, \sigma_{\mathrm{e}}(J)\right)^{1 / 2} \leq C \sum_{n=-\infty}^{\infty}\left|a_{n}-a_{n}^{(0)}\right|+\left|b_{n}-b_{n}^{(0)}\right| .
$$

\section{Remarks}

(1) The proof shows that $C$ can be chosen independently of the point on the isospectral torus of $\mathfrak{e}$.

(2) The proof works on $\left(\alpha_{1}-1, \alpha_{1}\right)$ and $\left(\beta_{\ell+1}, \beta_{j+1}+1\right)$, and then, using Proposition 4.5 , one gets bounds for $e \in\left(-\infty, \alpha_{1}\right) \cap \sigma(J)$ and for $e \in\left(\beta_{\ell+1}, \sigma\right) \cap \sigma(J)$, and since there are finitely many gaps, the following holds. 


\section{COROLLARY 6.2}

Under the hypotheses of Theorem 6.1,

$$
\sum_{e \in \sigma(J)} \operatorname{dist}\left(e, \sigma_{\mathrm{e}}(J)\right)^{1 / 2} \leq R H S \text { of }(6.1) .
$$

This then implies the following.

\section{Proof of Theorem 1.3}

Christiansen, Simon, and Zinchenko [18, Theorem 4.5] prove that (1.12) is implied by

$$
\begin{aligned}
& \text { (a) } \quad \text { LHS of }(6.2)<\infty, \\
& \text { (b) } \lim \left(\frac{a_{1} \cdots a_{n}}{C(\mathfrak{e})^{n}}\right) \text { exists in }(0, \infty) .
\end{aligned}
$$

Equation (6.3) follows from (1.11), (6.2), and an eigenvalue interlacing argument (since (6.2) is for full-line operators). (b) is immediate from $\sum_{n=1}^{\infty}\left|a_{n}-a_{n}^{(0)}\right|<\infty$ and the analogue of (6.4) for $a_{j}^{(0)}$ (see [18, Corollary 7.4]).

We prove Theorem 6.1 by showing the applicability of our Theorem 4.4. This requires the theory of eigenfunction expansions for one-dimensional absolutely continuous reflectionless systems and the theory of Jost functions for finite gap operators, where we follow the presentations of Breuer, Ryckman, and Simon [16] and Christiansen, Simon, and Zinchenko [17], respectively. We use their theorems but not their precise notation since there are conflicts between our notation in Section 4 and theirs.

We use $U_{n}^{ \pm}(\lambda)$ for the Weyl solutions of [16] at energy $\lambda$, defined for Lebesgue almost every $\lambda \in \sigma\left(J^{(0)}\right)$. They obey $J^{(0)} U^{ \pm}=\lambda U^{ \pm}$and are normalized by

$$
U_{0}^{ \pm}(\lambda)=1
$$

Since $J_{0}$ is reflectionless (see [49]), we have

$$
U_{n}^{-}=\overline{U_{n}^{+}},
$$

so the functions $f_{ \pm}(\lambda)$ of $[16,(2.4)]$ are equal with

$$
f_{ \pm}(\lambda)=-\left(4 \pi a_{0}\right)^{-1}\left(\operatorname{Im} U_{1}^{+}(\lambda)\right)^{-1},
$$

which we call $f$ below. Theorem 2.2 of [16] implies that if, for $\varphi_{n} \in \ell^{1} \cap \ell^{\infty}$,

$$
\widehat{\varphi}_{ \pm}(\lambda)=\sum_{n} \overline{U_{n}^{ \pm}(\lambda)} \varphi_{n}
$$


then

$$
\begin{gathered}
\varphi_{n}=\int\left[\widehat{\varphi}_{+}(\lambda) U_{n}^{+}(\lambda)+\widehat{\varphi}_{-}(\lambda) U_{n}^{-}(\lambda)\right] f(\lambda) d \lambda \\
\widehat{J \varphi_{ \pm}}(\lambda)=\lambda \widehat{\varphi}_{ \pm}(\lambda) \\
\left\|P_{a, b}\left(J_{0}\right) \varphi\right\|^{2}=\int_{a}^{b}\left(\left|\widehat{\varphi}_{+}(\lambda)\right|^{2}+\left|\widehat{\varphi}_{-}(\lambda)\right|^{2}\right) f(\lambda) d \lambda
\end{gathered}
$$

From [17], we need the covering map x: $\mathbb{C} \cup\{\infty\} \backslash \mathscr{L} \rightarrow \&$, where $\delta$ is the two-sheeted compact Riemann surface associated to the function

$$
D(x)=\left(\prod_{j=1}^{\ell+1}\left(x-\alpha_{j}\right)\left(x-\beta_{j}\right)\right)^{1 / 2} .
$$

$\mathscr{L}$, the limit set of a certain Fuchsian group, is a closed, nowhere dense, perfect subset of $\partial \mathbb{D}=\{z|| z \mid=1\}$. There is an open subset, $\mathcal{F} \subset \mathbb{D}$, on which $\mathbf{x}$ is one-to-one with $\mathbb{C} \cup\{\infty\} \backslash \mathfrak{e}$, whose closure is a fundamental domain for the Fuchsian group. For any band, $\left[\beta_{j}, \alpha_{j+1}\right]$, in $\mathbb{R} \backslash \mathfrak{e}$, there are $e^{i \varphi_{0}}, e^{i \varphi_{1}} \in \partial \mathbb{D}$ with $\varphi_{0}<\varphi_{1}$, so $\varphi \mapsto \mathbf{x}\left(e^{i \varphi}\right)$ maps $\left(\varphi_{0}, \varphi_{1}\right)$ bijectively onto the upper lip of the cut $\left(\beta_{j}, \alpha_{j+1}\right)$. What is crucial for us is that

$$
\frac{\partial \mathbf{x}\left(e^{i \varphi}\right)}{\partial \varphi} \neq 0, \quad \varphi \in\left(\varphi_{0}, \varphi_{1}\right), \quad \frac{\partial \mathbf{x}}{\partial \varphi}=0, \quad \frac{\partial^{2} \mathbf{x}}{\partial \varphi^{2}} \neq 0 \quad \text { at } \varphi_{0} \text { or } \varphi_{1} ;
$$

$\mathbf{x}$ is analytic in a neighborhood of $\left\{e^{i \varphi} \mid \varphi \in\left(\varphi_{0}, \varphi_{1}\right)\right\}$.

The fundamental Blaschke function, $B$, associated to $\mathbf{x}$ is a meromorphic function on $\mathbb{C} \cup\{\infty\} \backslash \mathscr{L}$, which is a Blaschke product and so obeys

$$
|z|<1 \Rightarrow|B(z)|<1, \quad|z|=1 \Rightarrow|B(z)|=1 .
$$

This, in turn, implies on $\partial \mathbb{D} \backslash \mathscr{L}$,

$$
B\left(e^{i \varphi}\right)=e^{i \tilde{\theta}(\varphi)}, \quad \frac{\partial \tilde{\theta}}{\partial \varphi}>0
$$

and $\tilde{\theta}$ is real analytic on $\partial \mathbb{D} \backslash \mathscr{L}$.

We let $\delta<\varphi_{1}-\varphi_{0}$ and define, for $k \in(-\delta, \delta)$,

$$
E(k)=\beta_{j}+\mathbf{x}\left(e^{i\left(\varphi_{0}+k\right)}\right)
$$


for $k \geq 0$ and $E(k)$ even. It is real analytic on $(-\delta, \delta)$ by (6.13). Define

$$
\theta(k)= \begin{cases}\tilde{\theta}\left(k+\varphi_{1}\right)-\tilde{\theta}\left(\varphi_{1}\right), & k>0, \\ -\theta(-k), & k<0,\end{cases}
$$

which is $C^{\infty}$ in $k$.

We let $\mathbb{G}$ denote the isospectral torus. There is a real analytic map $T: \mathbb{G} \rightarrow \mathbb{G}$ and a coordinate system on $\mathbb{G}$ in which $T$ is a group translation, and there are functions $A, B$ on $\mathbb{G}$ such that

$$
a_{n}(\vec{y})=A\left(T^{n} \vec{y}\right), \quad b_{n}(\vec{y})=B\left(T^{n} \vec{y}\right)
$$

for the Jacobi parameters for the Jacobi matrix $J^{(\vec{y})}$ with $\vec{y}$ in $\mathbb{G}$.

There are functions $\mathcal{f}(z ; \vec{y})$ (the Jost function) for $z \in \mathbb{C} \cup\{\infty\} \backslash \mathscr{L}, \vec{y} \in \mathbb{G}$ which are meromorphic in $z$, real analytic in $\vec{y}$, and whose only poles lie in $\mathbb{C} \cup\{\infty\} \backslash \overline{\mathbb{D}}$ with limit points only in $\mathscr{L}$. In particular, $\mathcal{L}$ is analytic, uniformly in $\vec{y}$, for $z$ in a neighborhood of $\left\{e^{i \varphi} \mid \varphi \in\left[\varphi_{0}, \varphi_{1}\right]\right\}$. The Jost solution is given by

$$
\mathscr{g}_{n}(z ; y)=a_{n}(y)^{-1} B(z)^{n} \mathcal{g}\left(z ; T^{n}(\vec{y})\right) .
$$

Suppose, for now, that the original Jacobi matrix, $J^{(0)}$, corresponding to $\vec{y}=0$, has

$$
g_{n=0}\left(e^{i \varphi_{0}} ; \vec{y}=0\right) \neq 0 .
$$

(Equivalently, $\mathcal{g}\left(e^{i \varphi_{0}} ; \vec{y}=0\right) \neq 0$.) $\mathcal{I}_{n}$ solves the difference equation $J^{(\vec{y})} \mathcal{g}_{n}(z ; y)=$ $x(z) \mathscr{I}_{n}(z ; y)$, so to get the normalization condition (6.5), we have

$$
U_{n}^{+}(\lambda)=\frac{\mathscr{I}_{n}(\mathbf{z}(\lambda) ; \vec{y}=0)}{\mathcal{J}_{0}(\mathbf{z}(\lambda) ; \vec{y}=0)}
$$

where $\mathbf{z}(\lambda)$ is determined by $\mathbf{x}(\mathbf{z}(\lambda))=\lambda$ with $\mathbf{z}(\lambda) \in\left\{e^{i \varphi} \mid \varphi_{0} \leq \varphi \leq \varphi_{1}\right\}$.

We define $\rho(k)$ by

$$
\rho(k)= \begin{cases}f y(E(k)) \frac{d}{d k} \mathbf{x}\left(e^{i\left(\varphi_{0}+k\right)}\right), & k \geq 0, \\ \rho(-k), & k<0 .\end{cases}
$$

We define $u_{n}^{+}(k)$ for $k \in(-\delta, \delta)$ by

$$
u_{n}^{+}(k)= \begin{cases}U_{n}^{+}(E(k)) \rho(k), & k>0, \\ U_{n}^{+}(E(k)) \rho(k), & k<0 .\end{cases}
$$


Finally,

$$
\tilde{\varphi}(k)= \begin{cases}\widehat{\varphi}_{+}(E(k)), & k \geq 0, \\ \widehat{\varphi}_{-}(E(k)), & k<0 .\end{cases}
$$

$\rho$ is picked to turn $f(\lambda) d \lambda$ in (6.11) to $\rho(k) d k$. It is then straightforward to check that (4.29) and (4.31) hold. Away from $k=0, \rho(k)$ is smooth, bounded, and nonvanishing. Since $u_{j}^{+}(k=0)=0, \operatorname{Im} u_{1}^{+}(k=0)=0$ and $f$ blows up there, but exactly as $1 / k\left[\left.\theta^{\prime}(k)\right|_{k=0}\right]$. Since $\frac{\partial \mathbf{x}}{\partial k}$ vanishes as $k$, by (6.13) $\rho$ has a smooth nonzero limit as $k \downarrow 0$; that is, (4.32) holds.

The relation (6.13) shows that at $k=0, E^{\prime}(k)=0, E^{\prime \prime}(k) \neq 0$, so (4.33) holds. Since $\mathcal{F}$ is uniformly bounded on $\mathbb{G}$ when $z \in\left\{e^{i \varphi} \mid \varphi_{0} \leq \varphi \leq \varphi_{1}\right\}$, (4.34) follows from (6.19).

$\theta$ is defined so that the $B(z)^{n}$ in (4.20) is replaced by $B\left(e^{i \varphi_{0}}\right)^{n}$ in the formula for $v$. Thus, $k$-derivatives are derivatives of $\mathcal{L}\left(e^{i\left(\varphi_{0}+k\right)}, T^{n}(\vec{y}=0)\right)$ which are bounded uniformly in $n$ by compactness of $\mathbb{G}$. First derivatives are zero and second derivatives are uniformly bounded in $n$ and $k \in(0, \delta)$, so (4.36) holds; (4.35) follows from (6.15). Thus, if (6.20) holds, Theorem 4.4 is applicable and proves Theorem 6.1.

Since nonzero solutions of a Jacobi eigenfunction equation cannot vanish at two successive points, if (6.20) fails for $\left\{a_{n}^{(0)}, b_{n}^{(0)}\right\}_{n=-\infty}^{\infty}$, it will not fail for $\left\{a_{n+1}^{(0)}, b_{n+1}^{(0)}\right\}_{n=-\infty}^{\infty}$, so we get Theorem 6.1 for a translated $J^{(0)}$. But since the conclusions are translation invariant, the theorem for the translated $J^{(0)}$ implies it for the original $J^{(0)}$.

Using the extensive literature on finite gap continuum Schrödinger operators (see Gesztesy and Holden [24] and references therein), it should be possible to prove a continuum analogue of the results of this section.

\section{Dirac equations}

Our decoupling results in Section 2 allow us to obtain some bounds on eigenvalues in the gap of one-dimensional Dirac operators. We do not require the results of Section 4. Let $\sigma_{1}, \sigma_{3}$ be the standard Pauli matrices, let $\sigma_{1}=\left(\begin{array}{ll}0 & 1 \\ 1 & 0\end{array}\right), \sigma_{3}=\left(\begin{array}{cc}1 & 0 \\ 0 & -1\end{array}\right), p=1 / i \frac{d}{d x}$ on $L^{2}(\mathbb{R}, d x)$, and let

$$
D_{0}=p \sigma_{1}+m \sigma_{3}=\left(\begin{array}{rr}
m & p \\
p & -m
\end{array}\right)
$$

be the free Dirac operator on $L^{2}\left(\mathbb{R}, \mathbb{C}^{2} ; d x\right)$. Here we prove the following.

THEOREM 7.1

Let $\gamma \geq 1 / 2$, and let $V \in L^{\gamma+1 / 2}(\mathbb{R}, d x) \cap L^{\gamma+1}(\mathbb{R}, d x)$. If $E_{j}$ denotes the eigen- 
values of $D_{0}+V$ in the gap $(-m, m)$, counting multiplicities, then

$$
\sum_{j}\left(m-\left|E_{j}\right|\right)^{\gamma} \leq C_{1, \gamma} \int_{\mathbb{R}}|V(x)|^{\gamma+1} d x+C_{2, \gamma} \sqrt{m} \int_{\mathbb{R}}|V(x)|^{\gamma+1 / 2} d x
$$

for some constants $C_{1, \gamma}, C_{2, \gamma}$ independent of $V$ and $m$.

The proof below yields explicit values of the constants.

The idea of the proof is to use Theorem 1.4 to reduce bounds to the scalar operators $\sqrt{p^{2}+m^{2}}-m-V_{ \pm}$and then to use Lieb-Thirring inequalities for $p^{2}-V_{ \pm}$and for $|p|-V_{ \pm}$to control $\sqrt{p^{2}+m^{2}}-m-V_{ \pm}$.

We recall the notation $S_{\gamma}$ from (1.2).

\section{THEOREM 7.2}

Let $\gamma>0$, and let $V \in L^{\gamma+1 / 2}(\mathbb{R}, d x) \cap L^{\gamma+1}(\mathbb{R})$. If $E_{j}$ denotes the eigenvalues of $D_{0}+V$ in $(-m, m)$, then

$$
\sum_{j}\left(m-\left|E_{j}\right|\right)^{\gamma} \leq 2\left[S_{\gamma}\left(H_{0}-V_{-}\right)+S_{\gamma}\left(H_{0}-V_{+}\right)\right],
$$

where $H_{0}$ is the operator $\sqrt{p^{2}+m^{2}}-m$ on $L^{2}(\mathbb{R}, d x)$.

We emphasize that we consider the operator $H_{0}$ acting on spinless (i.e., scalar) functions. One might wonder whether the inequality is true without the factor of 2.

\section{Proof}

By Theorem 1.4 and (4.16), one has

$$
\begin{aligned}
\sum_{j}\left(m-\left|E_{j}\right|\right)^{\gamma} & =\gamma \int_{0}^{m}(m-E)^{\gamma-1} N\left(D_{0}+V \in(-E, E)\right) d E \\
\leq \gamma \int_{0}^{m}(m-E)^{\gamma-1}( & N\left(V_{-}^{1 / 2}\left(D_{0}-E\right)^{-1} V_{-}^{1 / 2}>1\right) \\
& \left.+N\left(V_{+}^{1 / 2}\left(D_{0}+E\right)^{-1} V_{+}^{1 / 2}<-1\right)\right) d E .
\end{aligned}
$$

The $(2 \times 2)$-matrix, $\left(\begin{array}{cc}m-E & p \\ p & -m-E\end{array}\right)$, has eigenvalues $-E \pm \sqrt{p^{2}+m^{2}}$, which implies the operator inequalities

$$
\mp\left(D_{0} \pm E\right)^{-1} \leq\left(H_{0}+m-E\right)^{-1} \otimes I .
$$

Using this and the Birman-Schwinger principle, we find that

$$
\begin{aligned}
N\left(V_{-}^{1 / 2}\left(D_{0}-E\right)^{-1} V_{-}^{1 / 2}>1\right) & \leq 2 N\left(V_{-}^{1 / 2}\left(H_{0}+m-E\right)^{-1} V_{-}^{1 / 2}>1\right) \\
& =2 N\left(H_{0}-V_{-}<-m+E\right)
\end{aligned}
$$


and

$$
\begin{aligned}
N\left(V_{+}^{1 / 2}\left(D_{0}+E\right)^{-1} V_{+}^{1 / 2}<-1\right) & \leq 2 N\left(V_{+}^{1 / 2}\left(H_{0}+m-E\right)^{-1} V_{+}^{1 / 2}>1\right) \\
& =2 N\left(H_{0}-V_{+}<-m+E\right) .
\end{aligned}
$$

Plugging this into (7.4) and changing variables $\tau=m-E$, we obtain

$$
\sum_{j}\left(m-\left|E_{j}\right|\right)^{\gamma} \leq 2 \gamma \int_{0}^{m} \tau^{\gamma-1}\left(N\left(H_{0}-V_{-}<-\tau\right)+N\left(H_{0}-V_{+}<-\tau\right)\right) d \tau .
$$

Extending the integration to the whole interval $(0, \infty)$, we obtain $(7.3)$.

Theorem 7.1 follows immediately from Theorem 7.2 and Proposition 7.3 below. It relies on classical Lieb-Thirring bounds for $p^{2}+V$ and those for $|p|+V$ in the following form (see [20, Remark 4, p. 517] or [21, (13)]):

$$
\begin{array}{ll}
S_{\gamma}\left(p^{2}+V\right) \leq L_{\gamma} \int_{\mathbb{R}} V(x)_{-}^{\gamma+1 / 2} d x, & \gamma \geq \frac{1}{2}, \\
S_{\gamma}(|p|+V) \leq \tilde{L}_{\gamma} \int_{\mathbb{R}} V(x)_{-}^{\gamma+1} d x, & \gamma>0 .
\end{array}
$$

PROPOSITION 7.3

Let $\gamma \geq 1 / 2$, and let $0 \leq W \in L^{\gamma+1 / 2}(\mathbb{R}, d x) \cap L^{\gamma+1}(\mathbb{R})$. Then

$$
S_{\gamma}\left(H_{0}-W\right) \leq C_{1, \gamma} \int_{\mathbb{R}} W(x)^{\gamma+1} d x+C_{2, \gamma} \sqrt{m} \int_{\mathbb{R}} W(x)^{\gamma+1 / 2} d x
$$

for some constants $C_{1, \gamma}, C_{2, \gamma}$ independent of $W$ and $m$.

\section{Remark}

One could replace the right-hand side of (7.7) by a phase space bound.

\section{Proof}

Using the Birman-Schwinger principle, we write

$$
\begin{aligned}
S_{\gamma}\left(H_{0}-W\right) & =\gamma \int_{0}^{\infty} N\left(H_{0}-W \leq-\tau\right) \tau^{\gamma} d \tau \\
& =\gamma \int_{0}^{\infty} N\left(W^{1 / 2}\left(H_{0}-\tau\right)^{-1} W^{1 / 2}>1\right) \tau^{\gamma} d \tau .
\end{aligned}
$$

To estimate $N\left(W^{1 / 2}\left(H_{0}-\tau\right)^{-1} W^{1 / 2}>1\right)$, we fix two parameters, $0<\theta<1$ and $\rho>0$, and denote by $P$ and $P^{\perp}$ the spectral projections of $H$ onto the intervals 
$[0, \rho m)$ and $[m \rho, \infty)$, respectively. By Proposition 2.1,

$$
\begin{aligned}
N\left(W^{1 / 2}\left(H_{0}-\tau\right)^{-1} W^{1 / 2}>1\right) \leq N & \left(W^{1 / 2} P\left(H_{0}-\tau\right)^{-1} W^{1 / 2}>\theta\right) \\
& N\left(W^{1 / 2} P^{\perp}\left(H_{0}-\tau\right)^{-1} W^{1 / 2}>1-\theta\right) .
\end{aligned}
$$

There are constants, $c_{1}, c_{2}>0$, depending on $\rho$ such that

$$
\begin{aligned}
& \sqrt{p^{2}+m^{2}}-m \geq \frac{c_{1}}{m} p^{2} \quad \text { if }|p| \leq \rho m, \\
& \sqrt{p^{2}+m^{2}}-m \geq c_{2}|p| \quad \text { if } p \geq \rho m .
\end{aligned}
$$

Indeed, one can choose

$$
c_{1}=\frac{\sqrt{\rho^{2}+1}-1}{\rho^{2}}, \quad c_{2}=\frac{\sqrt{\rho^{2}+1}-1}{\rho} .
$$

This and the Birman-Schwinger principle yield

$$
\begin{aligned}
N\left(W^{1 / 2} P\left(H_{0}-\tau\right)^{-1} W^{1 / 2}>\theta\right) & \leq N\left(W^{1 / 2}\left(\frac{c_{1} p^{2}}{m}-\tau\right)^{-1} W^{1 / 2}>\theta\right) \\
& =N\left(\frac{c_{1} p^{2}}{m}-\theta^{-1} W<-\tau\right)
\end{aligned}
$$

and

$$
\begin{aligned}
N\left(W^{1 / 2} P^{\perp}(H-\tau)^{-1} W^{1 / 2}>1-\theta\right) & \leq N\left(W^{1 / 2}\left(c_{2}|p|-\tau\right)^{-1} W^{1 / 2}>1-\theta\right) \\
& =N\left(c_{2}|p|-(1-\theta)^{-1} W<-\tau\right) .
\end{aligned}
$$

Plugging this into (7.8) and doing the $\tau$-integration, we arrive at

$$
S_{\gamma}\left(H_{0}-w\right) \leq S_{\gamma}\left(\frac{c_{1} p^{2}}{m}-\theta^{-1} W\right)+S_{\gamma}\left(c_{2}|p|-(1-\theta)^{-1} W\right) .
$$

Using (7.5) and (7.6), we get

$$
\begin{aligned}
S_{\gamma}\left(H_{0}-W\right) \leq & c_{1}^{-1 / 2} \theta^{-\gamma-1 / 2} L_{\gamma} \sqrt{m} \int W^{\gamma+1 / 2} d x \\
& +c_{2}^{-1}(1-\theta)^{-\gamma-1} \tilde{L}_{\gamma} \int W^{\gamma+1} d x .
\end{aligned}
$$

This completes the proof of the proposition. 


\section{Appendix. Index theory proof of Proposition 2.3}

Here we provide a proof of Proposition 2.3 by using the theory of the index of a pair of orthogonal projections from [3]. This makes explicit the approach of Pushnitski [42] in his proofs of Proposition 2.3 and Theorem 1.4. Recall that if $P, Q$ are projections with

$$
\operatorname{dist}(P-Q, \text { compact operators })<1
$$

(and, in particular, if $P-Q$ is compact), one can define an integer index $(P, Q)$ by the equivalent definitions:

$$
\begin{aligned}
\operatorname{index}(P, Q) & =\operatorname{dim} \operatorname{ker}(P-Q-1)-\operatorname{dim} \operatorname{ker}(Q-P-1) \\
& =\operatorname{dim}\left(\operatorname{Ran} P \cap \operatorname{Ran} Q^{\perp}\right)-\operatorname{dim} \operatorname{ker}\left(\operatorname{Ran} Q \cap \operatorname{Ran} P^{\perp}\right) \\
& =\text { Fredholm index of } Q P \text { as a map of } \operatorname{Ran} P \text { to } \operatorname{Ran} Q .
\end{aligned}
$$

One has the following (see [3]).

(a) If $Q-R$ is compact, then

$$
\operatorname{index}(P, R)=\operatorname{index}(P, Q)+\operatorname{index}(Q, R)
$$

whenever (A.1) holds. This comes from (A.4), compactness of $P(Q-R) Q$, and invariance of the Fredholm index under compact perturbations.

(b) If $P-Q$ is of finite rank, then

$$
\text { index }(P, Q)=\operatorname{trace}(P-Q),
$$

and, in particular, if $P \geq Q$ also, so that $\operatorname{Ran} Q \subset \operatorname{Ran} P$, then

$$
\operatorname{index}(P, Q)=\operatorname{dim}\left(\operatorname{Ran} P \cap \operatorname{Ran} Q^{\perp}\right) .
$$

(c) If $Q(x)$ is norm continuous in $x$ for $x \in[a, b]$ and $Q(x)-P$ is compact for all such $x$, then

$$
\operatorname{index}(Q(b), P)=\operatorname{index}(Q(a), P) .
$$

(This follows from (A.5) and $\|Q(x)-Q(y)\|<1 \Rightarrow \operatorname{index}(Q(x), Q(y))=$ 0.)

Let $A$ be a self-adjoint operator bounded from below, and let $B$ be an $A$-form compact perturbation. Then for any $x_{0}$ and for $E_{0}$ sufficiently negative, $\left(A+x_{0} B-E_{0}\right)^{-1}-(A-$ $\left.E_{0}\right)^{-1}$ is compact, so by standard polynomial approximations, $f\left(A+x_{0} B\right)-f(A)$ is compact for all continuous $f$ of compact support. In particular, if $E \notin \sigma(A) \cup \sigma(A+$ 
$\left.x_{0} B\right)$, then $P_{(-\infty, E)}\left(A+x_{0} B\right)-P_{(-\infty, E)}(A)$ is compact and so has a relative index. Here is the key fact (a special case of Pushnitski [42, (2.12)]).

\section{PROPOSITION A.1}

Let $A$ be bounded from below, and let $B$ be a nonnegative form compact perturbation. Suppose $E \notin \sigma(A), \sigma(A+B)$ (resp., $\sigma(A), \sigma(A-B)$ ); then

$$
\operatorname{index}\left(P_{(-\infty, E)}(A+B), P_{(-\infty, E)}(A)\right)=-\delta_{+}(A, B ; E),
$$

respectively,

$$
\operatorname{index}\left(P_{(-\infty, E)}(A-B), P_{(-\infty, E)}(A)\right)=\delta_{-}(A, B ; E) \text {. }
$$

\section{Proof}

Since $\delta_{+}(A-B, B ; E)=\delta_{-}(A, B ; E)$ and $\operatorname{index}(P, Q)=-\operatorname{index}(Q, P)$, (A.9) implies (A.10), so we will prove that.

Let $x_{0} \in[0,1]$ be such that $E$ is an eigenvalue of $A+x_{0} B$ of multiplicity $k$. We show, for all sufficiently small $\varepsilon$, that

$$
\operatorname{index}\left(P_{(-\infty, E)}\left(A+\left(x_{0}+\varepsilon\right) B\right), P_{(-\infty, E)}\left(A+\left(x_{0}-\varepsilon\right) B\right)\right)=-k .
$$

Then, since $E$ is an eigenvalue of $A+x B$ for only finitely many $x$ 's and index $\left(P_{(-\infty, E)}(A+x B), P_{(-\infty, E)}(A)\right)$ is constant on the intervals between such $x$ 's (by (c) above), (A.11) implies (A.9).

Since $E \notin \sigma(A)$, there exists $\delta_{0}>0$, so $\left[E-\delta_{0}, E+\delta_{0}\right] \cap \sigma(A)=\emptyset$. Then for all $x, A+x B$ has only finitely many eigenvalues in $\left[E-\delta_{0}, E+\delta_{0}\right]$, and these eigenvalues are monotone in $x$. It follows that we can find $\varepsilon_{0}>0$, and then $0<\delta<\delta_{0}$ so that

(a) For $x \in\left(x_{0}-\varepsilon_{0}, x_{0}+\varepsilon_{0}\right), A+x B$ has exactly $k$ eigenvalues in $[E-\delta / 2, E+$ $\delta / 2]$ and no eigenvalues in $[E-\delta, E-\delta / 2) \cup(E+\delta / 2, E+\delta]$.

(b) If $x_{0}-\varepsilon_{0}<x<x_{0}$ (resp., $x_{0}<x<x_{0}+\varepsilon_{0}$ ), these $k$ eigenvalues are all in $[E-\delta / 2, E]$ (resp., $[E, E+\delta / 2])$.

If $0<\varepsilon<\varepsilon_{0}$, we have (the second and fourth follow from monotonicity, continuity, and (b))

$$
\begin{array}{r}
P_{(-\infty, E]}\left(A+\left(x_{0}-\varepsilon\right) B\right)=P_{(-\infty, E+\delta]}\left(A+\left(x_{0}-\varepsilon\right) B\right), \\
\text { index }\left(P_{(-\infty, E+\delta]}\left(A+\left(x_{0}-\varepsilon\right) B\right), P_{(-\infty, E+\delta]}\left(A+x_{0} B\right)\right)=0, \\
P_{(-\infty, E]}\left(A+\left(x_{0}+\varepsilon\right) B\right)=P_{(-\infty, E-\delta]}\left(A+\left(x_{0}+\varepsilon\right) B\right), \\
\text { index }\left(P_{(-\infty, E-\delta]}\left(A+\left(x_{0}+\varepsilon\right) B\right), P_{(-\infty, E-\delta]}\left(A+x_{0} B\right)\right)=0 .
\end{array}
$$


Thus, by (A.5),

$$
\begin{aligned}
\operatorname{LHS} \text { of }(\mathrm{A} .11) & =\operatorname{index}\left(P_{(-\infty, E-\delta]}\left(A+x_{0} B\right), P_{(-\infty, E+\delta]}\left(A+x_{0} B\right)\right) \\
& =-k
\end{aligned}
$$

by (A.6).

Proof of Proposition 2.3

By Proposition A.1 and (A.5), both sides of (2.4) are index $\left(P_{(-\infty, E)}(A), P_{(-\infty, E)}(A+\right.$ $\left.B_{+}-B_{-}\right)$).

Acknowledgments. We thank Alexander Pushnitski and Robert Seiringer for valuable discussions.

\section{References}

S. AlAMA, M. AVELLANEDA, P. A. DEIFT, and R. HEMPEL, On the existence of eigenvalues of a divergence-form operator $A+\lambda B$ in a gap of $\sigma(A)$, Asymptotic Anal. 8 (1994), 311 -344. MR 1278777464

2] ALAMA, P. A. DEIFT, and R. HEMPEL, Eigenvalue branches of the Schrödinger operator $H-\lambda W$ in a gap of $\sigma(H)$, Comm. Math. Phys. 121 (1989), $291-321$. MR 0985401464

J. AVRON, R. SEILER, and B. SIMON, The index of a pair of projections, J. Funct. Anal. 120 (1994), 220-237. MR 1262254 467, 488

J. AVRON and B. SIMON, The asymptotics of the gap in the Mathieu equation, Ann. Physics 134 (1981), 76-84. MR 0626698480

M. SH. BIRMAN, "Discrete spectrum in the gaps of the continuous one in the large-coupling-constant limit" in Order, Disorder and Chaos in Quantum Systems (Dubna, Russia, 1989), Oper. Theory Adv. Appl. 46, Birkhäuser, Basel, 1990, 17-25. MR 1124649464

6] - "Discrete spectrum in a gap of perturbed periodic operator at large coupling constants" in Rigorous Results in Quantum Dynamics (Liblice, Czech Rep., 1990), World Sci., River Edge, N.J., 1991, 16-24. MR 1243026

large coupling constant" in Estimates and Asymptotics for Discrete Spectra of Integral and Differential Equations (Leningrad, 1989-90), Adv. Soviet Math. 7, Amer. Math. Soc., Providence, 1991, 57-73. MR 1306508 Funct. Anal. Appl. 25 (1991), 158 - 161. MR 1142222 , "The discrete spectrum in gaps of the perturbed periodic Schrödinger operator, I: Regular perturbations" in Boundary Value Problems, Schrödinger Operators, Deformation Quantization, Math. Topics 8, Akademie, Berlin, 1995, 334-352. MR 1389015469 
[10] - The discrete spectrum of the periodic Schrödinger operator perturbed by a decreasing potential, St. Petersburg Math. J. 8 (1997), 1-14. MR 1392011

[11] - The discrete spectrum in gaps of the perturbed periodic Schrödinger operator, I: Nonregular perturbations, St. Petersburg Math. J. 9 (1998), 1073-1095. MR 1610239

[12] M. SH. BIRMAN, A. LAPTEV, and T. A. SUSLINA, The discrete spectrum of a two-dimensional second-order periodic elliptic operator perturbed by a decreasing potential, I: A semi-infinite gap, St. Petersburg Math. J. 12 (2001), 535-567. MR 1793617

[13] M. SH. BIRMAN and A. B. PUSHNITSKII, The discrete spectrum in the gaps of the perturbed pseudo-relativistic magnetic Hamiltonian, J. Math. Sci. (New York) 101 (2000), 3437 - 3447. MR 1698515

[14] M. SH. BIRMAN and G. D. RAĬKOV, "Discrete spectrum in the gaps for perturbations of the magnetic Schrödinger operator" in Estimates and Asymptotics for Discrete Spectra of Integral and Differential Equations (Leningrad, 1989-90), Adv. Soviet Math. 7, Amer. Math. Soc., Providence, 1991, 75-84. MR 1306509

[15] M. SH. BIRMAN and T. WEIDL, "The discrete spectrum in a gap of the continuous one for compact supported perturbations" in Mathematical Results in Quantum Mechanics (Blossin, Germany, 1993), Oper. Theory Adv. Appl. 70, Birkhäuser, Basel, 1994, 9-12. MR 1308999464

[16] J. BREUER, E. RYCKMAN, and B. SIMON, Equality of the spectral and dynamical definitions of reflection, Comm. Math. Phys. 295 (2010), 531 - 550.

MR 2594337481

[17] J. CHRISTIANSEN, B. SIMON, and M. ZINCHENKO, Finite gap Jacobi matrices, I: The isospectral torus, Constr. Approx. 32 (2010), 1 -65. MR 2659747 464, 481, 482

[18] - Finite gap Jacobi matrices, II: The Szegó class, Constr. Approx. (2010). DOI 10.1007/s00365-010-9094-7. 481

[19] D. DAMANIK, R. KILLIP, and B. SIMON, Perturbations of orthogonal polynomials with periodic recursion coefficients, Ann. of Math. (2) 171 (2010), 1931-2010. MR 2680401 462, 463

[20] I. DAUBECHIES, An uncertainty principle for fermions with generalized kinetic energy, Comm. Math. Phys. 90 (1983), 511-520. MR 0719431486

[21] R. L. FRANK, E. H. LIEB, and R. SEIRINGER, "Equivalence of Sobolev inequalities and Lieb-Thirring inequalities" in XVIth International Congress on Mathematical Physics (Prague, August, 2009), World Scientific, Singapore, 2010, 523-535. 486

[22] R. L. FRANK, B. SIMON, and T. WEIDL, Eigenvalue bounds for perturbations of Schrödinger operators and Jacobi matrices with regular ground states, Comm. Math. Phys. 282 (2008), 199-208. MR 2415477462

[23] F. GESZTESY, D. GURARIE, H. HOLDEN, M. KLAUS, L. SADUN, B. SIMON, and P. VOGL, Trapping and cascading of eigenvalues in the large coupling limit, Comm. Math. Phys. 118 (1988), 597-634. MR 0962490464 [24] F. GESZTESY and H. HOLDEN, Soliton Equations and Their Algebro-Geometric Solutions. Vol. I: $(1+1)$-Dimensional Continuous Models, Cambridge Stud. Adv. Math. 79, Cambridge Univ. Press, Cambridge, 2003. MR 1992536484 
[25] F. GESZTESY and B. SIMON, On a theorem of Deift and Hempel, Comm. Math. Phys. 116 (1988), 503-505. MR 0937772464

[26] R. HEMPEL, On the asymptotic distribution of the eigenvalue branches of the

Schrödinger operator $H \pm \lambda W$ in a spectral gap of $H$, J. Reine Angew. Math. 399 (1989), 38-59. MR 1004132

D. HUNDERTMARK, E. H. LIEB, and L. E. THOMAS, A sharp bound for an eigenvalue moment of the one-dimensional Schrödinger operator, Adv. Theor. Math. Phys. 2 (1998), 719-731. MR 1663336 462, 466, 472

D. HUNDERTMARK and B. SIMON, Lieb-Thirring inequalities for Jacobi matrices, J. Approx. Theory 118 (2002), 106-130. MR 1928259 462, 463, 476

- Eigenvalue bounds in the gaps of Schrödinger operators and Jacobi matrices, J. Math. Anal. Appl. 340 (2008), 892-900. MR 2390896464

R. KILLIP and B. SIMON, Sum rules for Jacobi matrices and their applications to spectral theory, Ann. of Math. (2) 158 (2003), 253-321. MR 1999923463

M. KLAUS, Some applications of the Birman-Schwinger principle, Helv. Phys. Acta 55 (1982/83), 49-68. MR 0674865464

F. KLOPP and J. RALSTON, "Endpoints of the spectrum of periodic operators are generically simple" in Cathleen Morawetz: A Great Mathematician, Methods Appl. Anal. 7, Int. Press, Somerville, Mass., 2000, 459-463. MR 1869296469 A. LAPTEV and T. WEIDL, "Recent results on Lieb-Thirring inequalities" in Journées "Équations aux Dérivées Partielles" (La Chapelle sur Erdre, France, 2000), Univ. Nantes Press, Nantes, 2000, exp. no. XX. MR 1775696462

S. Z. LEVENDORSKII, Lower bounds for the number of eigenvalue branches for the Schrödinger operator $H-\lambda W$ in a gap of $H$ : The case of indefinite $W$, Comm. Partial Differential Equations 20 (1995), 827-854. MR 1326908464 E. H. LIEB and W. THIRRING, "Inequalities for the moments of the eigenvalues of the Schrödinger Hamiltonian and their relation to Sobolev inequalities" in Studies in Mathematical Physics: Essays in Honor of Valentine Bargmann, Princeton Univ. Press, Princeton, 1976, 269-303. 462

W. MAGNUS and S. WINKLER, Hill's Equation, Intersci. Tracts in Pure and Appl. Math.

20, Intersci. Wiley New York, 1966. MR 0197830478

P. NEVAI, "Orthgonal polynomials, recurrences, Jacobi matrices, and measures" in Progress in Approximation Theory (Tampa, 1990), Springer Ser. Comput. Math.

19, Springer, New York, 1992, 79-104. MR 1240779463 
[42] A. PUSHNITSKI, Operator theoretic methods for the eigenvalue counting function in spectral gaps, Ann. Henri Poincaré 10 (2009), 793 - 822. MR 2520855 466, 467, 488, 489

[43] M. REED and B. SIMON, Methods of Modern Mathematical Physics, IV: Analysis of Operators, Academic Press, New York, 1978. MR 0493421 469, 478

[44] _ Methods of Modern Mathematical Physics, I: Functional Analysis, 2nd ed. Academic Press, New York, 1980, MR 0751952 464, 468

[45] O. L. SAFRONOV, The discrete spectrum in the gaps of the continuous one for non-signdefinite perturbations with a large coupling constant, Comm. Math. Phys. 193 (1998), 233-243. MR 1620254464

[46] - The discrete spectrum in the spectral gaps of semibounded operators with non-sign-definite perturbations, J. Math. Anal. Appl. 260 (2001), 641-652. MR 1845573

[47] - The discrete spectrum of self-adjoint operators under perturbations of variable sign, Comm. Partial Differential Equations 26 (2001), 629-649. MR 1842044464

[48] B. SIMON, Trace Ideals and Their Applications, 2nd ed., Math. Surveys Monog. 120, Amer. Math. Soc., Providence, 2005. MR 2154153469

[49] - Szegô's Theorem and Its Descendants: Spectral Theory for $L^{2}$ Perturbations of Orthogonal Polynomials, Princeton Univ. Press, Princeton, 2011. 463, 481

[50] A. V. SOBOLEV, On the asymptotics of the discrete spectrum in gaps of the continuous spectrum of the perturbed Hill operator, Funct. Anal. Appl. 25 (1991), 162 - 164. MR 1142223464

[51] _ _Weyl asymptotics for the discrete spectrum of the perturbed Hill operator" in Estimates and Asymptotics for Discrete Spectra of Integral and Differential Equations (Leningrad, 1989-90), Adv. Soviet Math. 7, Amer. Math. Soc., Providence, 1991, 159-178. MR 1306512 464, 471

[52] _ _ "Recent results on the Bethe-Sommerfeld conjecture" in Spectral Theory and Mathematical Physics: A Festschrift in Honor of Barry Simon's 60th Birthday, Proc. Sympos. Pure Math. 76, Part 1, Amer. Math. Soc., Providence, 2007, 383-398. MR 2310211469

[53] F. TRÈVES, Topological Vector Spaces, Distributions and Kernels, unabridged republication of the 1967 original, Dover, Mineola, N.Y., 2006. MR 2296978 473

[54] T. WEIDL, On the Lieb-Thirring constants $L_{\gamma, 1}$ for $\gamma \geq 1 / 2$, Comm. Math. Phys. 178 (1996), 135-146. MR 1387945 462, 466

\section{Frank}

Department of Mathematics, Princeton University, Princeton, New Jersey 08544, USA; rlfrank@math.princeton.edu

\section{Simon}

Department of Mathematics, California Institute of Technology, Pasadena, California 91125, USA; bsimon@caltech.edu 